\title{
A GEOMETRICAL OBSERVATION ON THE ARF INVARIANT OF A FRAMED MANIFOLD
}

\author{
NIGEL RAY \\ 1. Introduction
}

The Arf (or Kervaire) invariant of a framed manifold is a framed bordism invariant defined in dimensions $4 k+2, k \geqslant 0$. In the cases $k=0,1,3$ and 7 non-zero Arf invariants arise from awkward framings, rather than awkward manifolds. This prompts

(1.1) Conjecture. (See [1]). Each framed bordism class contains a manifold which admits $a$ (possibly different) framing with zero Arf invariant.

In this note we remark that a recent result of D. S. Kahn and S. B. Priddy implies that the stable $J$-homomorphism is epic in positive dimensions (modulo odd torsion), and then explain how this in turn establishes the truth of (1.1).

These observations owe much to conversations with $M$. G. Barratt and R. Switzer.

\section{Bordism groups}

Using the Pontrjagin-Thom construction, we may identify the bordism ring of framed manifolds $(M, \phi)$ with $S_{*}$, the stable homotopy ring of spheres. More generally, the unreduced framed bordism group of a space $X$ consists of framed bordism classes of triples $(M, \phi ; f)$, where $f$ is a map $M \rightarrow X$. We may then set up an isomorphism of this group with $S_{*}\left(X_{+}\right)$, the stable homotopy of $X$ with disjoint basepoint (see [3]).

Now consider the biframed bordism ring $\Omega_{*}^{b i}$, defined by biframed bordism classes of triples $\left(M, \phi_{1}, \phi_{2}\right)$ where $\phi_{1}$ and $\phi_{2}$ are framings of $M$. Let $O$ denote the infinite orthogonal group, so that $S_{*}\left(O_{+}\right)$is a Pontrjagin ring.

(2.1) Proposition.

$$
S_{*}\left(O_{+}\right) \cong \Omega_{*}^{b i}
$$

Proof. Given a framed manifold $(M, \phi)$, a map $f: M \rightarrow O$ may be used to alter $\phi$ to a second framing $f \phi$. Thus we have a function $(M, \phi ; f) \mapsto(M, \phi, f \phi)$.

Conversely, if $M$ has framings $\phi_{1}$ and $\phi_{2}$ there is a map $\phi_{2} / \phi_{1}: M \rightarrow O$ which measures their difference. So we have a second function $\left(M, \phi_{1}, \phi_{2}\right) \mapsto\left(M, \phi_{1} ; \phi_{2} / \phi_{1}\right)$ and it is simple to check that these two are mutually inverse homomorphisms of bordism classes.

Received 16 July, 1971; revised 11 December, 1971.

[Bull. LoNdon MATH. Soc., 4 (1972), 163-164] 
Since the difference map of a product of biframed manifolds is the Whitney sum of the respective difference maps, our isomorphism is of rings.

The reduced framed bordism group of a space $X$ is the subgroup of $S_{*}\left(X_{+}\right)$ given by triples $(M, \phi ; f)$ for which $(M, \phi)$ represents zero in $S_{*}$. The generalised Pontrjagin-Thom construction identifies this subgroup with $S_{*}(X)$, the stable homotopy of $X$.

In this language, the stable $J$-homomorphism $J^{S}: S_{*}(O) \rightarrow S_{*}$ factors as the inclusion $S_{*}(O)<\Omega_{*}^{b i}$ followed by the map $\Omega_{*}^{b i} \rightarrow S_{*}$ which forgets the first framing.

So we have

(2.2) Lemma. (1.1) is true if $J^{s}: S_{*}(0) \rightarrow S_{*}$ is epic (modulo odd torsion) for $n>0$.

Note that if $J^{S}$ is epic, it furnishes a positive solution to the following:

(2.3) CONJECTURE. Each framed bordism class contains a manifold which can be reframed so as to be a framed boundary.

\section{Application of a theorem of Kahn and Priddy}

The $J$-homomorphism is induced by a standard map $O(n) \rightarrow \Omega^{n} S^{n}$. We can compose this with the embedding of real projective space $R P^{n-1} \subset O(n)$ to obtain $g_{n}: R P^{n-1} \rightarrow \Omega^{n} S^{n}$. By taking the adjoint of $g_{n}$ and letting $n \rightarrow \infty$, we induce a composition $S_{*}\left(R P^{\infty}\right) \rightarrow S_{*}(O) \underset{J_{s}}{\rightarrow} S_{*}$ to which we may apply

(3.1) THEOREM (Kahn and Priddy [2]). The homomorphism $S_{n}\left(R P^{\infty}\right) \rightarrow S_{n}$ is epic (modulo odd torsion) for $n>0$.

(3.2) Corollary. The same is true for $J^{S}$.

So by $(2.2)$ we have proved that conjecture (1.1) is true. Also, we have established (2.3) modulo odd torsion, and shown that the reframing involved has a particularly simple nature.

\section{References}

1. M. G. Barratt, Conjecture 22, Conference on Algebraic Topology (University of 1llinois at Chicago Circle, June, 1968).

2. D. S. Kahn and S. B. Priddy, Applications of the transfer to stable homotopy theory (Preprint, Northwestern Univ.).

3. R. E. Stong, Notes on Cobordism theory (Princeton Univ. Press, 1968).

The University, Manchester. 